\title{
Case report: fainting during acupuncture stimulation at acupuncture point LI4
}

\author{
O sang Kwon, Kwang-Ho Choi, Junbeom Kim, Seong Jin Cho, Suk-Yun Kang, Ji-Young Moon and Yeon Hee Ryu ${ }^{*}$
}

\begin{abstract}
Background: Fainting is one of the major adverse events that can occur as a result of acupuncture treatment. However, the observation of changes in biological parameters is rarely available when fainting occurs. In this case report, we could observe changes in the electroencephalogram (EEG) in a participant who fainted while participating in a clinical trial aiming to observe a relationship between acupuncture stimulation at LI4 acupuncture point and EEG in healthy adults.

Case presentation: The EEG pattern of participant changed twice. The first change was in response to the acupuncture needle insertion, and the second change occurred during fainting. Both changes consisted of a burst in EEG amplitude, but the pattern of details was different. Multiple areas of the cortex were activated, and the increased ratio of the $\gamma$ wave was not observed during fainting. While acupuncture needle insertion, only the sensory cortex were activated and increased the ratio of the $\gamma$ wave.

Conclusions: This single case is presented to improve the understanding of fainting during acupuncture as an adverse event and to explore the mechanism of acupuncture treatment, despite the absence of statistics and repeatability. This information can provide a new viewpoint about the mechanism of acupuncture treatment and the possibility of new techniques based on acupuncture.
\end{abstract}

Keyword: Fainting during acupuncture, Acupuncture, Fainting, EEG, sLoreta

\section{Background}

The efficacy, economical feasibility and safety of acupuncture treatment have been demonstrated in thousands of clinical studies. Acupuncture treatment can replace or reduce the dosage of the drugs such as amitriptyline [1], taxane [2] or morphine [3] to prevent adverse event that are prescribed to patients to treat various disease including chronic pain. This replacement reduces the adverse effects of drugs, however, acupuncture treatment may also be associated with minor and less critical adverse effects.

Pain and bleeding are reviewed as the most common adverse events (AEs) of acupuncture treatment from 73 case reports and 14 case series [4]. In addition to these mild AEs, fainting, stroke, haemorrhage or traumatic injuries were reported as serious AEs and led to death in some cases [4]. However, a more recent survey indicated that acupuncture clinics and the potential of severe AEs are rare [5].

* Correspondence: yhryu@kiom.re.kr

KM Fundamental Research Division, Korea Institute of Oriental Medicine, Daejeon, Korea
Fainting is a common AE which contains dizziness, perspiration and syncope as a symptom [6], during acupuncture treatment and has not been classified as a critical $\mathrm{AE}$ because fainting does not kill the patient or have long-term effects, although it does cause some patients to avoid future acupuncture treatments because of the traumatic experience. However, some researchers have proposed that fainting can be used to access the mechanism of acupuncture treatment in the brain. The mechanism and cause of fainting during acupuncture treatment have not been closely examined because researchers cannot induce participants to faint.

This case occurred as an adverse event during a clinical trial designed to observe changes in EEG patterns affected by acupuncture treatment. EEG signals were recorded in the pre-stage before acupuncture needle insertion, and recording was discontinued when the participant fell down. The EEG pattern during fainting was collected until the participant fall down, and we present the data of this single case herein. 


\author{
Case presentation \\ Participant information \\ Age: 20 \\ Gender: female \\ Height: $160.0 \mathrm{~cm}$ \\ Weight: $41.0 \mathrm{~kg}$ \\ Body temperature: $37.4{ }^{\circ} \mathrm{C}$ \\ Average blood pressure: 106/61 $\mathrm{mmHg}$ \\ Marriage: none \\ Job: student (college) \\ Diet: regular \\ Exercise: none \\ Smoking: none \\ Drinking: 0.5 bottle (beer)/week \\ Specific disease: none \\ Medical history: no specific medical history
}

\section{Adverse event}

Participant were participated in the clinical trial to observe the effect of acupuncture stimulation at the electroencephalogram (EEG) and peripheral nerve system in male and female adults at the Daejeon Korean medicine hospital of Daejeon university (KCT0001871). The adverse event occurred during visit 2 (2015.05.20). The participant arrived at $10 \mathrm{am}$, and her behaviour and language were normal. The participant was prepared for measurements with a multichannel EEG (ActiveTwo, BIOSEMI, Netherland) and was placed in a seated position on a chair. Electrodes were attached, and the strength of the electrical stimulation was calibrated. A sterilised single-use acupuncture needle $(0.3 \times 30 \mathrm{~mm}$, Dongbang medical, Korea) was inserted into the acupuncture point large intestine 4 (LI4, Hapgok) of right hand of participant approximately in depth of $1.8 \mathrm{~cm}$ by licensed KMD who has a 10 year's career and 4 year of the career was as a assistant teacher of acupuncture practice at the university, after $30 \mathrm{~min}$ of rest. The EEG data were recorded for approximately $5 \mathrm{~min}$ before the acupuncture needle was inserted, and researchers noticed the fainting approximately $4 \mathrm{~min}$ after needle insertion. The participant did not report any symptoms or uncomfortable feelings. The researchers heard the sound of some objects falling down and saw participant falling at that time. The EEG electrodes were separated from the participant's body due to the impact of falling down.

\section{Subsequent observations}

We placed the participant on the bed in a supine position. The participant recovered before $1 \mathrm{~min}$ had passed. The participant drank a cup of warm water and rested for 30 min while lying on the bed.

The participant's condition was observed at both 7 and 30 days after fainting. The participant did not report any uncomfortable feeling or poor condition. She also did not show any traumatic reaction or negative opinion about acupuncture treatment and was willing to receive acupuncture treatment again.

During the first interview, the participant admitted that she had been on a diet while participating in the test.

\section{Analysis}

Frequency analysis using Fourier transformation was performed to quantitatively analyse the changes in EEG activity. A window of $2 \mathrm{~s}$ (75\% sliding window) was applied to the full-time data. The ratio was normalized as the ratio of the total power at each window after adding the absolute power of each frequency band $(\varepsilon: 5-8 \mathrm{~Hz}$, $\alpha$ : 9-12 Hz, $\beta$ : 13-30 Hz, $\gamma$ : over $30 \mathrm{~Hz}$ ).

Standard low-resonance electromagnetic tomography analysis (sLoreta) [7, 8] was performed using a LORETA-KEY program available at: http://www.uzh.ch/ keyinst/loreta.htm.

\section{EEG wavelength amplitude}

The absolute power of the EEG signal increased twice during the procedure. The first burst was observed after the acupuncture needle was inserted (300 s). The next burst was observed after the participant fainted (540 s). An increase in the $\gamma$ wave was observed (Fig. 1). The ratios of the $\varepsilon, \alpha, \beta$ and $\gamma$ wave bands showed a specific pattern at the same time of the bursts. The ratios of the $\varepsilon$ and $\gamma$ waves increased significantly, and the ratio of the $\beta$ wave decreased significantly (Figs. 2 and 3).

\section{EEG activity in the brain}

EEG activity in the brain was analysed using the Brodmann area (BA) [9]. The orbitofrontal cortex (BA11) was activated before acupuncture treatment. The medial part of the primary sensory cortex (BA3, 1 and 2) and the somatosensory association cortex (BA5) were activated after the acupuncture needle was inserted. While the patient was fainting, the EEG signal was activated at various areas: the dorsolateral prefrontal cortex (DLPFC, BA46,9) or insula cortex (BA16); pars orbitalis (BA 47); part of the secondary visual cortex (V2, BA18) and the associative visual cortex (V3,V4,V5, BA19); and the middle temporal gyrus (BA21).

\section{Discussion and Conclusion}

Fainting during acupuncture treatment is one of the most common adverse effects that occurs at the acupuncture clinic [4] and is believed by some researchers to be related to the mechanisms of acupuncture treatment. However, studies on fainting during acupuncture have not been performed because of ethical and technical issues. Thus, fainting during acupuncture in a clinical trial, particularly a brain imaging study, can be a great opportunity to observe what happens as 


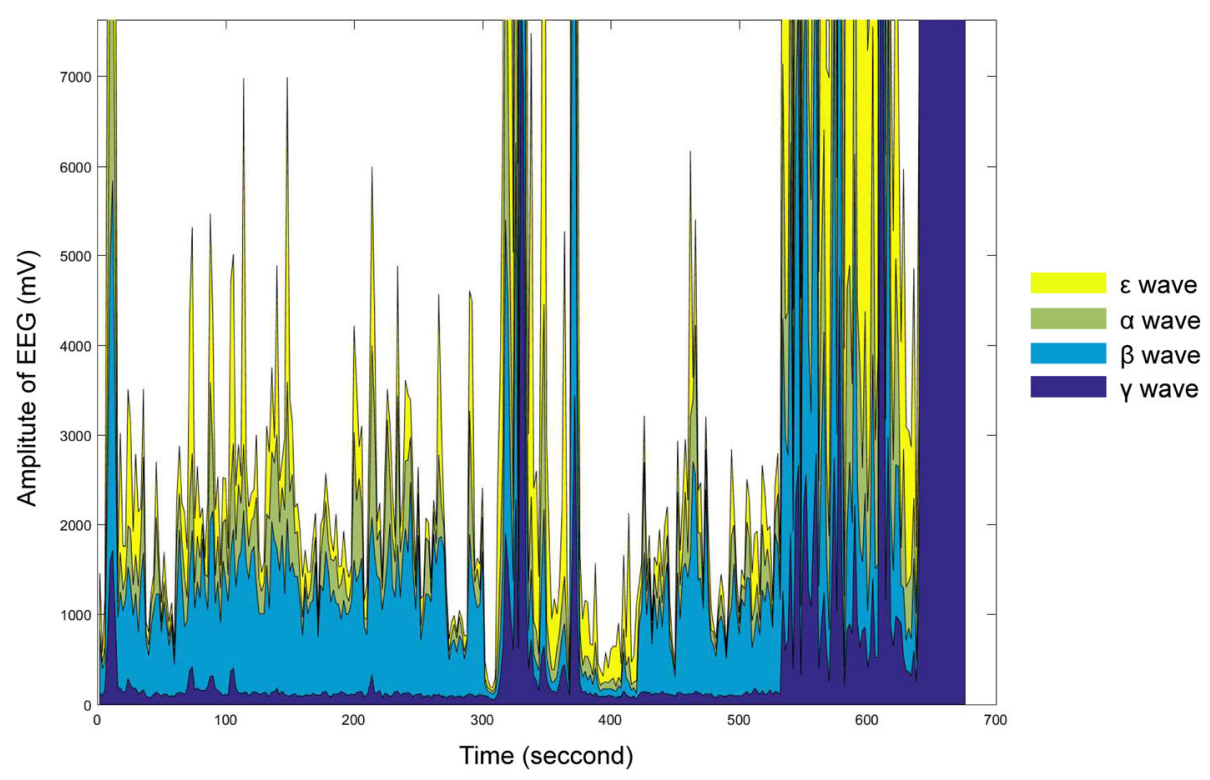

Fig. 1 Changes in the absolute power of EEG bands. The acupuncture needle was inserted at approximately $300 \mathrm{~s}$ (red vertical line), and fainting was observed at approximately $540 \mathrm{~s}$. The EEG amplitude rapidly increased twice: once at the time of needle insertion and once at the time of fainting

fainting occurs. In this study, we had the opportunity to observe EEG patterns during fainting and present the data herein to enlarge our experience and understanding the relationship between the brain and acupuncture.

Before acupuncture needle injection, the amplitude of the EEG did not show a specific pattern. EEG activity was detected in the orbitofrontal cortex (BA11), which is known to be related to the reward system [10]. We hypothesized that the patient was anxious and afraid of acupuncture following her interview; this was her first experience with acupuncture.

The absolute power of the EEG signal significantly increased twice, and the patterns of the two bursts were different. The ratio and absolute power of the $\gamma$ wave, which is related to the somatosensory [11] area and perception [12], increased significantly and appeared to modulate the stimulation from the acupuncture needle insertion. This analysis was supported by sLoreta location analysis, which showed EEG activity in the medial

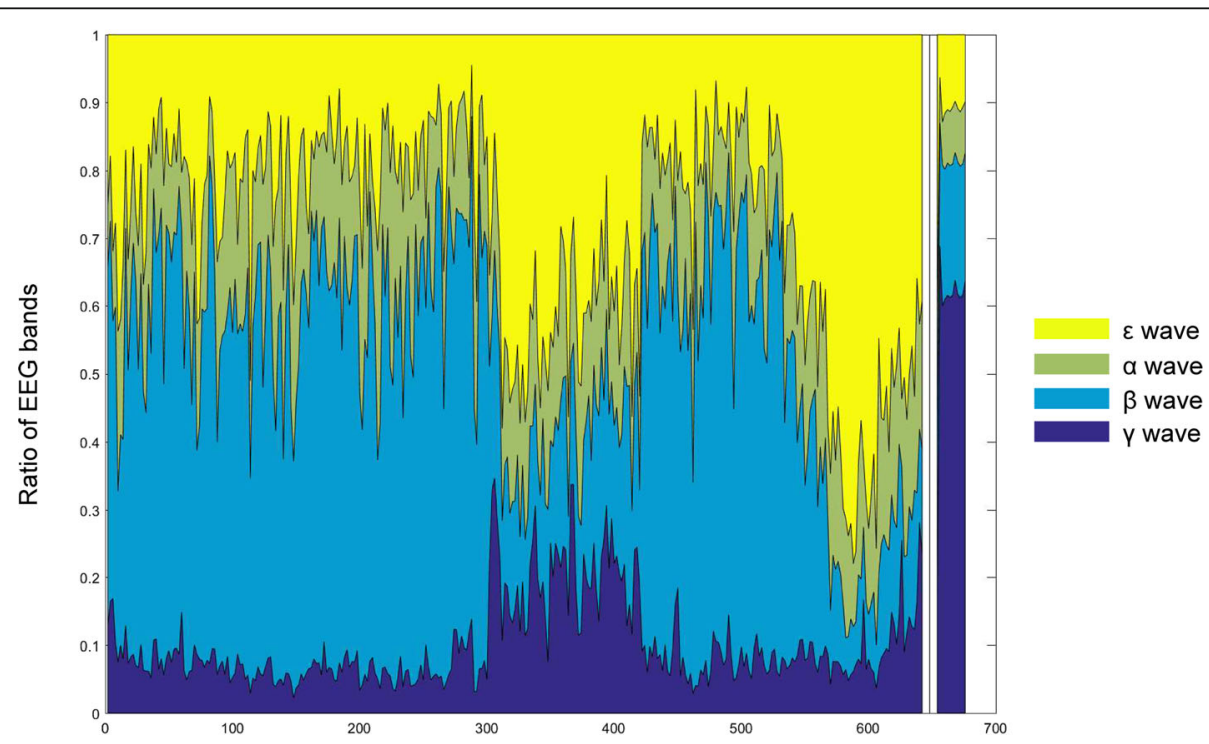

Fig. 2 Changes in ratio between each EEG band. Both activity bursts showed an increase in the $\varepsilon$ wave ratio. The ratio of the $y$ wave did not significantly increase when the patient fainted, but it did increase significantly when the acupuncture needle was inserted 


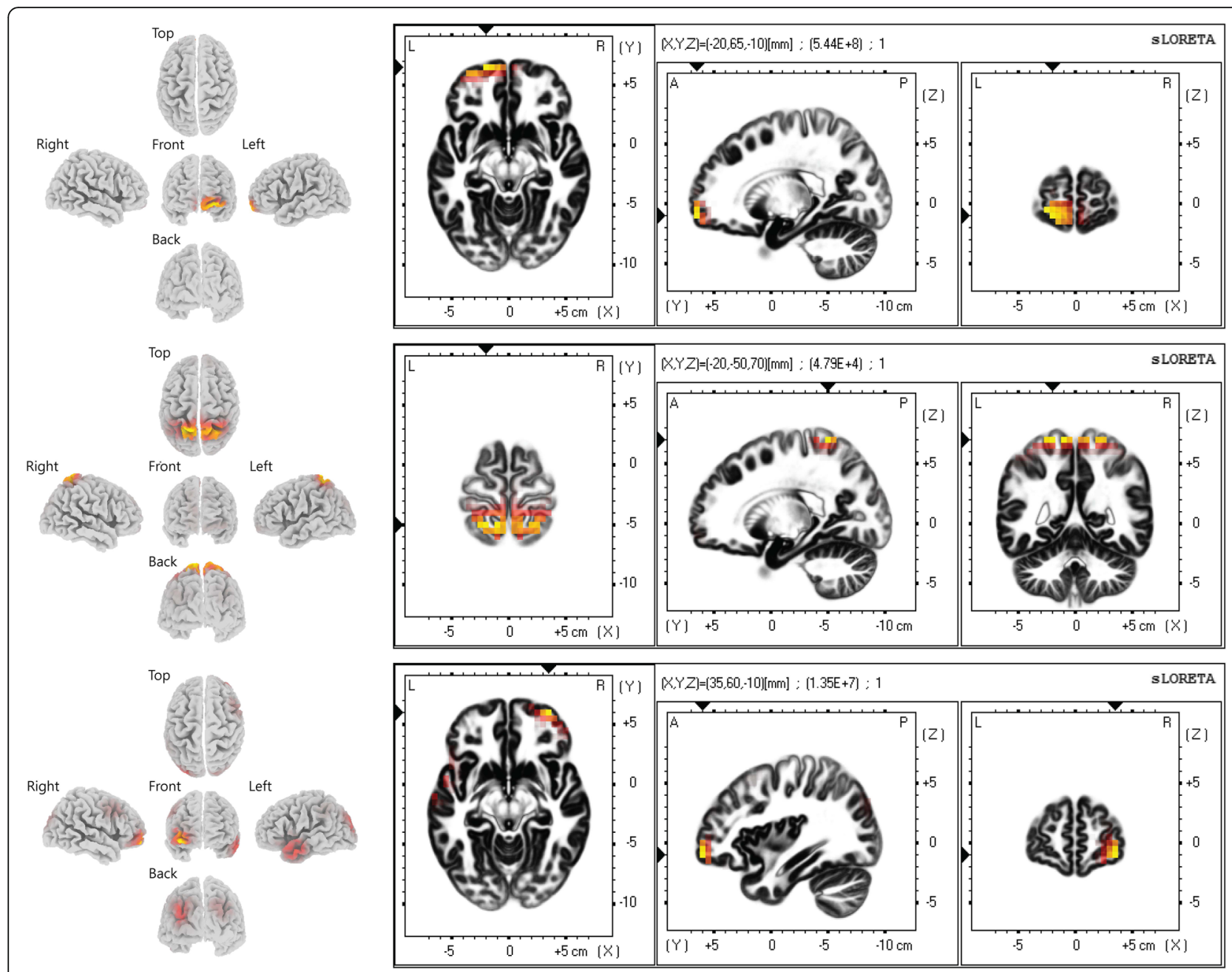

Fig. 3 Cortical areas activated at each stage. Coloured spaces indicate an increase in neuronal activity. The yellow colour indicates a more active area than a red-coloured area. Upper: baseline activity before acupuncture needle insertion. Middle: the first burst when the acupuncture needle inserted; the sensory cortex was activated. Lower: the second burst when the participant fainted; activation occurred in various areas

part of the primary sensory cortex (BA3, 1 and 2) and the somatosensory association cortex (BA5), thus which indicating stimulation of the forearm or hand. The increase of EEG amplitude was observed at prior studies $[13,14]$ and even very significantly increasing of the amplitude also observed [15], however, in some study, we could find that most of channels are not changed [1].

The second burst showed a different pattern. The ratio and absolute power of the $\gamma$ wave did not increase when the burst started, whereas the first burst showed an increase in the $\gamma$ wave at the start of the burst. The sLoreta analysis also indicated different locations of activation. The amplitude of the brain waves increased at various locations of cortex, such as the DLPFC, the insula cortex, the pars orbitalis, part of the secondary/associative visual cortex and the middle temporal gyrus. Activation of the DLPFC and the insula cortex during acupuncture treatment has been reported by various researchers $[16,17]$. However, the other areas were not reported to have a major role and did not have any relationship with the efficacy area of LI4. Thus, we considered that additional excitation at these areas may provoke fainting, although the mechanism and connection between these area is not clear.

In the later interview, participant reported that she had never experienced fainting or syncope. Participant's average systolic/diastolic blood pressure before the test was 106/61 and we concluded it as a normal range and not enough for fainting regarding her body mass index. She reported she was not fasted although she was trying to lose her weight. There was no patients of epilepsy, amyotrophic lateral sclerosis or other genetic neural disease in her family according herself and she had never experienced fainting before the test in accordance with her statement. We also asked the participant to contact us when she experience fainting or similar situation 
again and we could get any message from her for more than one and half years. We concluded that her physical statement such as blood pressure, body weight or etc. stayed at the low but normal range and may work without any problem before, however, those statement may affected her to faint when we induced acupuncture stimulation.

This case is the first reported case of EEG changes observed while fainting caused by acupuncture stimulation and comparison or statistical analysis could not performed. Thus, this one case cannot reveal the parameters or mechanisms of fainting during acupuncture treatment. However, fainting cannot be intentionally induced by acupuncture, and this accidental case is considered to be the first report about what occurs in the brain when people faint during acupuncture treatment.

\section{Abbreviations}

AEs: Adverse events; BA: Brodmann area; EEG: Electroencephalogram; sLoreta: Standard low-resonance electromagnetic tomography analysis

\section{Acknowledgement}

This study was funded by Korea Institute of Oriental Medicine (KIOM) (grant no. K16070).

\section{Funding}

This study was funded by Korea Institute of Oriental Medicine (KIOM) (grant no. K16070).

\section{Availability of data and materials}

Not applicable. If your manuscript does not contain any data, please state not applicable in this section.

\section{Authors' contributions}

$\mathrm{KO}$ mainly analysed and wrote script text, $\mathrm{K}-\mathrm{HC}$ and JK analysed EEG data, CSJ edited paragraphs, KS-Y and MJ-Y analysed brain areas, RYH corrected data analysis and manuscript text. All authors read and approved the final manuscript.

\section{Competing interest}

The authors declare that they have no competing interests.

\section{Consent for publication}

This article does not contain any individual person's data which let to identify the participant's identification. And also, written consent about publication of the data has signed by participant.

\section{Ethics approval and consent to participate}

This study performed at the Daejeon Korean medicine hospital of Daejeon university under the review of the Oriental Hospital of DaeJeon University IRB. The study protocol has registered to the clinical research information service (KCT0001871).

Received: 1 September 2016 Accepted: 2 March 2017

Published online: 09 March 2017

\section{References}

1. Choi K-H, Kwon OS, Cho SJ, Lee S, Kang S-Y, Ahn SH, Ryu Y. Evaluating acupuncture point and nonacupuncture point stimulation with EEG: a highfrequency power spectrum analysis. Evid Based Complement Altern Med. 2016;2016:2134364.

2. Greenlee H, Crew KD, Capodice J, Awad D, Buono D, Shi Z, Jeffres A, Wyse S, Whitman W, Trivedi MS. Randomized sham-controlled pilot trial of weekly electro-acupuncture for the prevention of taxane-induced peripheral neuropathy in women with early stage breast cancer. Breast Cancer Res Treat. 2016;156(3):453-64
3. Gwak YS, Kim HY, Lee BH, Yang CH. Combined approaches for the relief of spinal cord injury-induced neuropathic pain. Complement Ther Med. 2016;25:27-33.

4. Zhang J, Shang H, Gao X, Ernst E. Acupuncture-related adverse events: a systematic review of the Chinese literature. Bull World Health Organ. 2010; 88(12):915-21.

5. Ernst E, White AR. Prospective studies of the safety of acupuncture: a systematic review. Am J Med. 2001;110(6):481-5.

6. Association TKAaM. The acupuncture and moxibustion. In: Jipmundang 2008. p. 474-5.

7. Pascual-Marqui RD. Standardized low-resolution brain electromagnetic tomography (sLORETA): technical details. Methods Find Exp Clin Pharmacol. 2002;24(Suppl D):5-12.

8. Pascual-Marqui RD. Discrete, 3D distributed, linear imaging methods of electric neuronal activity. Part 1: exact, zero error localization. arXiv preprint arXiv:07103341 2007

9. Brodmann K. Brodmann's: Localisation in the cerebral cortex. Springer Science \& Business Media 2007.

10. Rogers RD, Owen AM, Middleton HC, Williams EJ, Pickard JD, Sahakian BJ, Robbins TW. Choosing between small, likely rewards and large, unlikely rewards activates inferior and orbital prefrontal cortex. J Neurosci. 1999; 19(20):9029-38.

11. Cardin JA, Carlen M, Meletis K, Knoblich U, Zhang F, Deisseroth K, Tsai L-H, Moore $\mathrm{Cl}$. Driving fast-spiking cells induces gamma rhythm and controls sensory responses. Nature. 2009;459(7247):663-7.

12. Fries P. Neuronal gamma-band synchronization as a fundamental process in cortical computation. Annu Rev Neurosci. 2009;32(1):209-24.

13. Pei X, Wang J, Deng B, Wei X, Yu H. WLPVG approach to the analysis of EEG-based functional brain network under manual acupuncture. Cogn Neurodyn. 2014;8(5):417-28.

14. Kim MS, Kim HD, Seo HD, Sawada K, Ishida M. The effect of acupuncture at PC-6 on the electroencephalogram and electrocardiogram. Am J Chin Med. 2008:36(03):481-91.

15. Sakai S, Hori E, Umeno K, Kitabayashi N, Ono T, Nishijo H. Specific acupuncture sensation correlates with EEGs and autonomic changes in human subjects. Auton Neurosci. 2007;133(2):158-69.

16. Chae Y, Lee I-S, Jung W-M, Park K, Park H-J, Wallraven C. Psychophysical and neurophysiological responses to acupuncture stimulation to incorporated rubber hand. Neurosci Lett. 2015;591:48-52.

17. Napadow V, Dhond RP, Kim J, LaCount L, Vangel M, Harris RE, Kettner N, Park K. Brain encoding of acupuncture sensation - coupling on-line rating with fMRI. Neuro Image. 2009;47(3):1055-65.
Submit your next manuscript to BioMed Central and we will help you at every step:

- We accept pre-submission inquiries

- Our selector tool helps you to find the most relevant journal

- We provide round the clock customer support

- Convenient online submission

- Thorough peer review

- Inclusion in PubMed and all major indexing services

- Maximum visibility for your research

Submit your manuscript at www.biomedcentral.com/submit
Biomed Central 